\title{
Review: studies of different interventions have mixed results for psychosocial outcomes after stroke
}

Knapp P, Young J, House A, et al. Non-drug strategies to resolve psycho-social difficulties after stroke. Age Ageing 2000

Jan;29:23-30.

QUESTION: In patients with stroke, what interventions improve psychosocial outcomes?

\section{Data sources}

Studies were identified by searching Medline, EMBASE/ Excerpta Medica, PsycLIT, the Cochrane Library, and secondary references and by consulting experts in the field.

\section{Study selection}

Studies were selected if they were randomised controlled trials (RCTs) that assessed primarily the improvement of psychosocial outcomes. Studies of physical rehabilitation were excluded.

\section{Data extraction}

Data were extracted on study objectives, patient characteristics, length of follow up, type of intervention, and results. The methodological quality of the studies and their reporting were assessed (concealment of allocation, blinded assessment of outcomes, and predefined sample size).

\section{Main results}

Information provision and education: 1 RCT showed that an information leaflet increased patients' knowledge about their illness and prognosis at follow up but not their knowledge about services and benefits. 2 RCTs showed that information booklets did not increase patients' social activities, knowledge about their illness, or satisfaction with care. 1 RCT supplemented an information pack with a 1 hour visit from a nurse, which led to a decrease in depression scores at 6 months but had no effect on anxiety or caregiver outcomes. A 2 hour education programme during the first month after stroke improved caregiver knowledge of stroke and several aspects of family coping.

Leisure therapy: 1 RCT found that 5 visits $(7 \mathrm{~h}$ total $) \geq 1$ year after stroke led to an increased frequency and duration of leisure activities. 1 RCT found no effect for 5 visits shortly after hospital discharge, but the comparison group received a similar amount of contact, which may have confounded results.

Support workers: a stroke family care worker who helped to identify and fulfil unmet patient and carer needs led to small differences between the treatment and control group. Improvements in social adjustment, depression, and helplessness were seen in patients in the control group, whereas service satisfaction was greater in patients in the treatment group. Carers in the treatment group had improved mood and greater service satisfaction than those in the control group. 1 RCT evaluated an intervention in which nurses visited patients and carers at home to provide support and help with problem solving. Groups did not differ for mood or social activities in patients or caregivers. Mildly disabled patients showed a modest improvement in social activities. Caregivers reported improved support, and they valued the service. 2 RCTs evaluated contact with a single social worker; 1 RCT found no effect on independence or social function, and the other RCT found a high level of patient satisfaction with the social work service but no effect on patient or carer mood.
Counselling: in 1 RCT, cognitive behaviour therapy (in addition to educational classes) improved patients' adjustment to stroke. The use of Egan's problem solving approach in $\leq 7$ sessions led to a decrease in depression scores of patient and carers but mood did not differ otherwise (1 RCT). 1 RCT attempted to study the improvement of social networks but both groups had preexisting robust social networks and no difference between groups was seen.

\section{Conclusion}

In patients with stroke, the evidence involves different approaches, and results for psychosocial outcomes are mixed.

\section{COMMENTARY}

Stroke is a major cause of morbidity and mortality, and the high prevalence of psychosocial difficulties after stroke substantially impairs recovery and increases long term mortality. ${ }^{1}$ Stroke rehabilitation research is often based on small single centre trials, with negative or weakly positive results, ${ }^{2}$ and it has been difficult to differentiate between the effect of a specific intervention and the natural recovery process. ${ }^{3}$

Knapp et al summarise the selected RCTs of psychosocial interventions after stroke in a table and described them in the text according to the nature of the experimental intervention. Although the number of studies was adequate, the data were not combined or evaluated formally "because of the heterogeneity of interventions and outcome measures used." The differences in findings were, as the authors suggested, most likely attributable to the differences in the nature, type, and delivery of the interventions.

The review suggested that currently limited evidence exists for the effectiveness of psychosocial interventions after stroke. The review was not truly systematic, ${ }^{45}$ however, and does not therefore contribute evidence in support of that notion.

The clinician may draw some benefit from the information that specific types of strategies in certain groups appeared to be effective, but no convincing biomedical evidence can be gleaned from the studies. The clinician should also note that the review highlights the need for coordinated, collaborative clinical research in the area.

The authors rightly suggest that future trials should be undertaken with greater attention to quality of design and implementation.

David Burke, BMed, FRANZCP New South Wales Institute of Psychiatry Sydney, Australia

1 Wade D. Rehabilitation therapy after stroke. Lancet 1999;354:176-7.

2 Gladman J, Barer D, Langhorne P. Specialist rehabilitation after stroke. $B M J$ 1996;312:1623-4.

3 Helgason CM, Wolf PA. American Heart Association Prevention Conference IV: prevention and rehabilitation of stroke: executive summary. Circulation 1997;96:701-7.

4 Mulrow CD. Rationale for systematic reviews. BMJ 1994;309:597-9.

5 Liddle J, Williamson M, Irwig L. Method for evaluating research and guideline evidence. Sydney: NSW Health Department, 1996. 